\title{
Support Estimation of a Sample Space-Time Covariance Matrix
}

\author{
Connor Delaosa ${ }^{1}$, Jennifer Pestana ${ }^{2}$, Nicholas J. Goddard ${ }^{3}$, Samuel D. Somasundaram ${ }^{4}$, and Stephan Weiss ${ }^{1}$ \\ ${ }^{1}$ Dept. Electronic \& Electrical Engineering, University of Strathclyde, Glasgow G1 XW, Scotland \\ ${ }^{2}$ Dept. Mathematics \& Statistics, University of Strathclyde, Glasgow G1 XW, Scotland \\ ${ }^{3}$ Dstl Portsdown West, Fareham, Hampshire PO17 6AD, UK \\ ${ }^{4}$ Thales Underwater Systems, Cheadle, Stockport, UK \\ \{connor.delaosa,jennifer.pestana,stephan.weiss\}@strath.ac.uk
}

\begin{abstract}
The ensemble-optimum support for a sample spacetime covariance matrix can be determined from the ground truth space-time covariance, and the variance of the estimator. In this paper we provide approximations that permit the estimation of the sample-optimum support from the estimate itself, given a suitable detection threshold. In simulations, we provide some insight into the (in)sensitivity and dependencies of this threshold.
\end{abstract}

Index Terms-space-time covariance matrix, parahermitian matrix, cross-correlation sequence, estimation.

\section{INTRODUCTION}

The space-time covariance matrix of a vector of $M$ sensor measurements $\mathbf{x}[n] \in \mathbb{C}^{M}, \mathbf{R}[\tau]=\mathcal{E}\left\{\mathbf{x}[n] \mathbf{x}^{\mathrm{H}}[n-\tau]\right\}$, where $\mathcal{E}\{\cdot\}$ is the expectation operator, represents the data's second order statistics, and is therefore central in the formulation of many broadband array processing problems. This includes for example broadband MIMO systems [1], coding [2], beamforming [3], [4], source separation [5], angle of arrival estimation [6], scene discovery [7], and many others applications. Based on factorisations of its $z$-transform $\boldsymbol{R}(z)=\sum_{\tau} \mathbf{R}[\tau] z^{-\tau}$ such as the polynomial eigenvalue (EVD) [8]-[10], or singular value decompositions [8], [11], well-known narrowband optimal solutions [12], [13] can be directly extended to the broadband case.

For any of the above applications, the space-time covariance matrix needs to be estimated from the measurements $\mathbf{x}[n]$, and typically for a limited set of snapshots, say for $n=0 \ldots(N-1)$, either due to the limited availability of data, or the need to restrict the estimation to an interval over which the data can be assumed to be stationary. While various investigations have been undertaken into the accuracy of the above decompositions [14], [15] and limiting factors due to algorithm-internal order reductions [16]-[19] or the conditioning of the underlying source model [20], it is only recently that the estimation errors of $\mathbf{R}[\tau]$ [21] and their impact on the factorisation of the parahermitian matrix EVD of $\boldsymbol{R}(z)$ [22] have been investigated.

In the estimation of a sample covariance matrix $\hat{\mathbf{R}}[\tau]$ from a limited set of data, it is important to know over which support

This work was supported in parts by the Engineering and Physical Sciences Research Council (EPSRC) Grant number EP/S000631/1 and the MOD University Defence Research Collaboration in Signal Processing, Dstl, and a John Anderson Research Award by the University of Strathclyde. this estimate should be calculated as this direct impacts on the error propagation and computational complexity of any subsequent processing. As a byproduct of the analysis of the variance of $\hat{\mathbf{R}}[\tau]$, in [21], an optimum support was derived based on the knowledge of $\mathbf{R}[\tau]$. Since the ground truth statistics are inaccessible in practise, in this paper we aim to estimate the optimum support from the data over a given sample set.

\section{SAmple Space-Time Covariance Matrix ESTIMATION}

Since the space-time covariance matrix is constructed of cross-correlation sequences, its estimation reduces to the estimation of these quantities. This section provides an overview of the estimator and its variance by summarising results from [21], [22].

\section{A. Unbiased Estimator}

Assuming ergodicity for two stationary signals $x_{m}[n]$ and $x_{\mu}[n], m, \mu \in\{1 \ldots M\}$, their cross-correlation sequence $r_{m \mu}[\tau]$ is defined as

$$
r_{m \mu}[\tau]=\mathcal{E}\left\{x_{m}[n] x_{\mu}^{*}[n-\tau]\right\} .
$$

An estimator for this quantity is

$$
\hat{r}_{m \mu}[\tau]= \begin{cases}\frac{1}{N-\tau} \sum_{n=0}^{N-\tau-1} x_{m}[n+\tau] x_{\mu}^{*}[n], & \tau \geq 0 \\ \frac{1}{N+\tau} \sum_{n=0}^{N+\tau-1} x_{m}[n] x_{\mu}^{*}[n-\tau], & \tau<0,\end{cases}
$$

when applied to $N$ snapshots in time of $x_{m}[n]$ and $x_{\mu}[n]$ for $n=0 \ldots(N-1)$. It is straightforward to show that the estimator in (2) is unbiased, and for example for $\tau \geq 0$,

$$
\begin{aligned}
\operatorname{mean}\left\{\hat{r}_{m \mu}[\tau]\right\} & =\mathcal{E}\left\{\hat{r}_{m \mu}[\tau]\right\} \\
& =\frac{1}{N-\tau} \sum_{n=0}^{N-\tau-1} \mathcal{E}\left\{x_{m}[n] x_{\mu}^{*}[n-\tau]\right\} \\
& =\frac{1}{N-\tau} \sum_{n=0}^{N-\tau-1} r_{m \mu}[\tau]=r_{m \mu}[\tau],
\end{aligned}
$$

i.e. the estimated quantity indeed tends towards the crosscorrelation sequence in (1).

Example. For the estimation of a cross-correlation produced by mixing matrices of order 30 based on the source model 


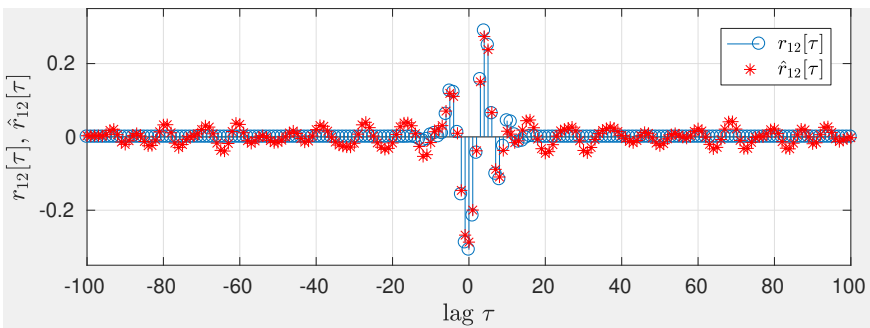

Fig. 1. example for a cross-correlation sequence $r_{12}[\tau]$ and its estimate $\hat{r}_{12}[\tau]$ based on $N=10^{3}$ snapshots of data.

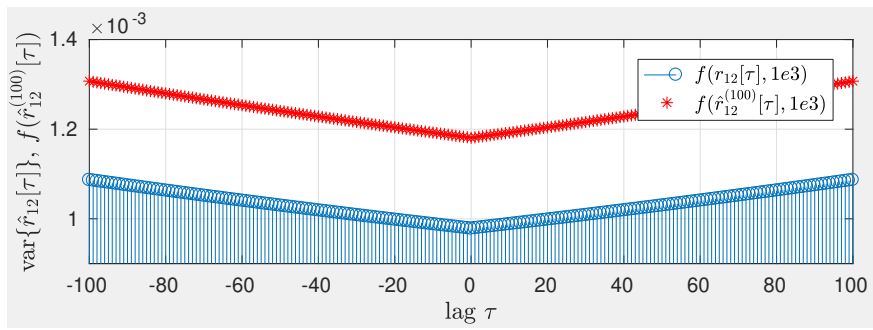

Fig. 2. Variance of the sample cross-correlation estimate shown in Fig. 1, and an approximation based on a sample estimate $\hat{r}_{12}[\tau]$ for $|\tau| \leq 100$.

in [23], Fig. 1 shows both the cross-correlation sequence $r_{12}[\tau]$ and its estimate $\hat{r}_{12}[\tau]$ for $|\tau| \leq 100$ using $N=10^{3}$ snapshots of data.

\section{B. Variance of Estimation}

The mismatch between the cross-correlation sequence in (1) and its estimate in (2), as illustrated in the example of Fig. 1, can be assessed by determining the variance of the estimator. In [21], this has been derived as

$$
\begin{aligned}
\operatorname{var}\left\{\hat{r}_{m \mu}[\tau]\right\}= & \frac{1}{(N-|\tau|)^{2}} \sum_{t=-N+|\tau|+1}^{N-|\tau|-1}(N-|\tau|-|t|) . \\
& \cdot\left(r_{m m}[t] r_{\mu \mu}^{*}[t]+\bar{r}_{m \mu}[\tau+t] \bar{r}_{m \mu}^{*}[\tau-t]\right)
\end{aligned}
$$

for a sample size $N$, where $\bar{r}_{m \mu}^{*}[\tau-t]=\mathcal{E}\left\{x_{m}[n] x_{\mu}[n-\tau]\right\}$ is the complementary cross-correlation, which vanishes if the data is complex-valued, circularly symmetric [24].

Example. For the cross-correlation sequence in Fig. 1 and a sample size of $N=10^{3}$ snapshots of complex-valued, circularly symmetric data, Fig. 2 shows the evaluation of $\operatorname{var}\left\{\hat{r}_{m \mu}[\tau]\right\}$ according to (3) for $|\tau| \leq 100$.

\section{Modelling Error}

Using the above derivations for the variance of its constituent components, the variance of the sample space-time covariance estimate now becomes the target. The space-time covariance matrix $\mathbf{R}[\tau]$ is assumed to have a support of length $2 \tau_{\max }+1$, i.e. $\mathbf{R}[\tau]=\mathbf{0} \forall|\tau|>\tau_{\max }$. With $\hat{\mathbf{R}}[\tau]$ evaluated over a support length of $2 T+1$, we write its mismatch as

$$
\mathbf{E}[\tau]=\mathbf{R}[\tau]-\hat{\mathbf{R}}[\tau],
$$

and define a mean square error, termed the ensemble modelling error, as

$$
\begin{aligned}
\xi & =\mathcal{E}\left\{\sum_{\tau=-\infty}^{\infty}\|\mathbf{E}[\tau]\|_{\mathrm{F}}^{2}\right\} \\
& =\underbrace{\sum_{\tau=-T}^{T} \mathcal{E}\left\{\|\mathbf{E}[\tau]\|_{\mathrm{F}}^{2}\right\}}_{\xi_{1}}+\underbrace{2 \sum_{\tau=T+1}^{\sum_{\max }}\|\mathbf{R}[\tau]\|_{\mathrm{F}}^{2}}_{\xi_{2}} .
\end{aligned}
$$

Of the two terms in (5), the first, $\xi_{1}$, is an estimation error due to (3), and the second, $\xi_{2}$, forms a truncation error with $\xi_{2}=0$ for $T \geq \tau_{\max }$.

Based on (3), the ensemble estimation error $\xi_{1}$ can be written as

$$
\xi_{1}=\sum_{\tau=-T}^{T} \sum_{m, \mu=1}^{M} \operatorname{var}\left\{\hat{r}_{m \mu}[\tau]\right\}
$$

Therefore, the modelling error $\xi$ depends only on the spacetime covariance matrix and its complement, the sample size $N$, and the support of the estimate, $T$.

\section{Ensemble-Optimum Support}

The ensemble estimation error in (6) represents the expected mean square value of the error $\boldsymbol{E}(z) \bullet-\circ \mathbf{E}[\tau]$ in (4), which is directly linked to the perturbation of the eigenvalues and eigenvectors of the parahermitian matrix EVD of $\boldsymbol{R}(z)$ when based on a sample estimate $\hat{\boldsymbol{R}}(z)$ [22]. With knowledge of the exact $\boldsymbol{R}(z)$ and the sample size $N$, the support providing the minimum mean squared modelling error across the ensemble is given by

$$
T_{\text {ensemble, opt }}=\arg \min _{T} \xi .
$$

In [21], $T_{\text {ensemble, opt }}$ has been explored as a trade-off between the terms $\xi_{1}$ and $\xi_{2}$. With $\xi_{2}=0$ for $T>\tau_{\max }$ and $\xi_{1}$ growing with increasing $T, T_{\text {ensemble, opt }}<\tau_{\max }$, i.e. generally the ensemble-optimum support of the estimate will be shorter than the support of $\mathbf{R}[\tau]$.

\section{SUPPORT ESTIMATION}

\section{A. Preliminary Consideration}

To find a practical tool for determining the support of a sample space-time covariance matrix, the ensemble-optimum support in (7) raises two issues. Firstly, $\mathbf{R}[\tau]$ is not known and secondly, $\xi$ and $\xi_{1}$ in (5) are ensemble averages. For an individual sample space-time covariance matrix estimate $\hat{\mathbf{R}}[\tau]$, its modelling error is $\hat{\xi}=\hat{\xi}_{1}+\xi_{2}$, such that $\xi_{1}=\mathcal{E}\left\{\hat{\xi}_{1}\right\}$. As a result, for an individual ensemble probe, we can generally find a better local support $T_{\text {opt }}$ obtained via

$$
T_{\text {opt }}=\arg \min _{T} \hat{\xi} .
$$

While $T_{\text {opt }}$ will be based on the knowledge of both $\mathbf{R}[\tau]$ and the specific estimate $\hat{\mathbf{R}}[\tau]$, below we aim to find an approximation, $\hat{T}_{\mathrm{opt}}$ that is solely based on $\hat{\mathbf{R}}[\tau]$. 


\section{B. Idea}

If (3) is defined as an operation

$$
\operatorname{var}\left\{\hat{r}_{m \mu}[\tau]\right\}=f\left(r_{m \mu}[\tau], N\right)
$$

and $\hat{r}_{m \mu}^{(T)}[\tau]$ is a sample estimate with support $2 T+1$, such that $\hat{r}_{m \mu}^{(T)}[\tau]=0 \forall|\tau|>T$ is assumed, then we find that for sufficiently large $T$, (9) can be very roughly approximated by

$$
f\left(\hat{r}_{m \mu}^{(T)}[\tau], N\right) \sim f\left(r_{m \mu}[\tau], N\right)
$$

i.e. instead of the inaccessible ground truth, the variance of the estimation can be based on the sample estimate itself.

Example. As empirical evidence, when using $r_{12}[\tau]$ and its sample estimate $\hat{r}_{12}^{(100)}[\tau]$ from Fig. 1, in addition to $\operatorname{var}\left\{\hat{r}_{12}[\tau]\right\}=f\left(r_{12}[\tau], N\right)$, Fig. 2 also shows $f\left(\hat{r}_{12}^{(100)}[\tau], N\right)$, which behaves very similarly, and therefore can provide a rough approximation to $\operatorname{var}\left\{\hat{r}_{12}[\tau]\right\}$.

Since the ensemble estimation error, $\xi_{1}$, can be calculated from $\operatorname{var}\left\{\hat{r}_{m \mu}[\tau]\right\}$ according to (6), it can now also roughly be based on $f\left(\hat{r}_{m \mu}^{(T)}[\tau], N\right)$. Instead of focusing on the overall estimation error, we only consider the error within a tail section of the estimate,

$$
\chi_{1}[T]=\sum_{m, \mu} \sum_{\tau=T+1}^{T+T_{0}} f\left(\hat{r}_{m \mu}^{(T)}[\tau], N\right)
$$

Note that $\chi_{1}[T]$ roughly approximates an estimation error portion for an estimate of larger support, $\hat{r}_{m \mu}^{\left(T+T_{0}\right)}[\tau]$, measured over the range $T \leq|\tau| \leq T+T_{0}$.

If we consider the energy in the tail section of an estimate $r_{m \mu}^{\left(T+T_{0}\right)}[\tau]$ of increased support, then

$$
\chi_{2}[T]=\sum_{m, \mu} \sum_{\tau=T+1}^{T+T_{0}}\left|r_{m \mu}^{\left(T+T_{0}\right)}[\tau]\right|^{2}
$$

will contain a portion of the estimation error, but also include a term that would otherwise have been truncated in $\hat{r}_{m \mu}^{(T)}[\tau]$ if $T$ was too short. Therefore the comparison of $\chi_{1}[T]$ and $\chi_{2}[T]$ enables us to detect if a truncation error has been incurred, in the case of the support $T$ of $\hat{r}_{m \mu}^{(T)}[\tau]$ being too restrictive.

\section{Hypothesis Test and Ratio of Variances}

The reasoning in Sec. III-B leads to the hypotheses

$$
\begin{array}{ll}
H_{0}: & \chi_{1}[T] \sim \chi_{2}[T] \\
H_{1}: & \chi_{1}[T] \ll \chi_{2}[T],
\end{array}
$$

i.e. $H_{0}$ hypothesises that only an estimation error is incurred, while $H_{1}$ indicates an additional truncation error. A value $T$ at the boundary between $H_{0}$ and $H_{1}$ can then provide an estimate $\hat{T}_{\text {opt }}$ for the optimum support $T_{\text {opt }}$ in (8).

To find the boundary between the hypotheses, and therefore $\hat{T}_{\text {opt }}$, here the behaviour of the ratio $\gamma[T]=\chi_{2}[T] / \chi_{1}[T]$ is inspected. Thus, $H_{0}$ is accepted for $\gamma[T] \sim 1$, while $H_{1}$ requires $\gamma[T] \gg 1$.
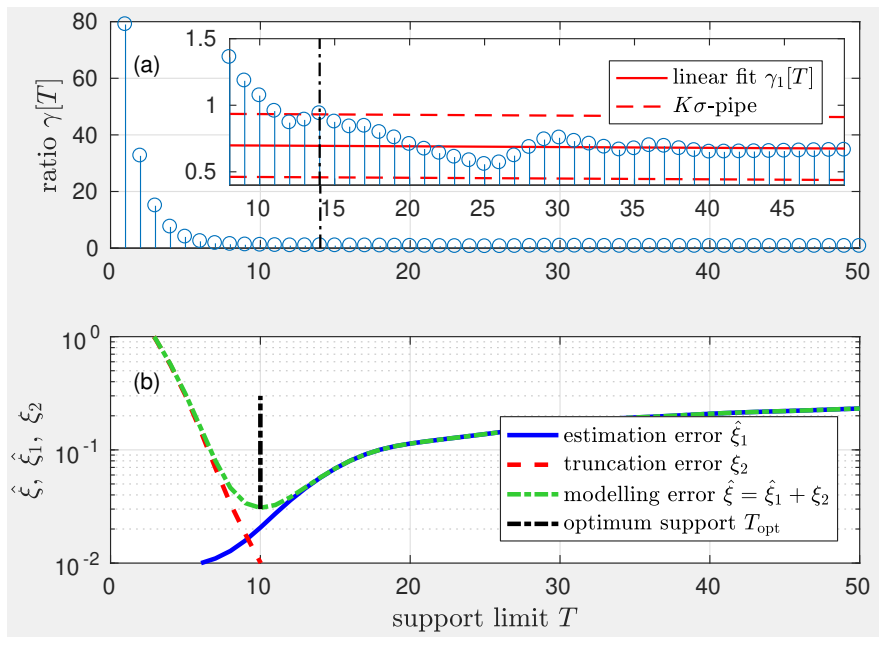

Fig. 3(a) ratio with threshold selection by a $K \sigma$-pipe based on a single sample set, and (b) optimum support based on ground truth $\mathbf{R}[\tau]$ and estimate $\hat{\mathbf{R}}[\tau]$ with support $2 T+1$.

\section{Threshold Determination}

Given the often rough approximations made in Sec. III-B, the selection of a suitable threshold in determining the switch between the two hypotheses in Sec. III-C is key. The proposed approach is based on inspecting $\gamma[T]$ for sufficiently high values of $T$, say beyond a value $T_{1}$, which are bound to exceed the support of $\mathbf{R}[\tau]$, or at least ensure that any elements of $\mathbf{R}[\tau]$ will be small compared to an estimation error. Over a range of values $T_{1} \leq \tau \leq 2 T_{1}$, we determine (i) a linear least squares fit $\gamma_{1}[T]$ to $\gamma[T]$, such that $\sum_{T=T_{1}}^{2 T_{1}}\left|\gamma[T]-\gamma_{1}[T]\right|^{2}$ is minimised, and (ii) the standard deviation $\sigma$ of $\gamma[T]$ w.r.t. this linear fit $\gamma_{1}[T]$. We then check whether for smaller values of $T$, i.e. $T<T_{1}$, values of $\gamma[T]$ fall outside a pipe of width $K \sigma$ around this linear fit, and estimate the optimum support as

$$
\hat{T}_{\mathrm{opt}}=\arg \max _{T} T \quad \text { s.t. } \gamma[T]>\gamma_{1}[T]+K \sigma,
$$

with $K$ yet to be determined.

Example. The approach is exemplified in Fig. 3(a), where $T_{1}=50$, and $\gamma[T]$ as well as the linear fit with a pipe of width $K \sigma$, here for $K=5$, are shown. In this case, $\hat{T}_{\text {opt }}=14$. With exact knowledge of $\mathbf{R}[\tau]$, and given the sample estimate $\hat{\mathbf{R}}[\tau]$, we can exactly determine the modelling and truncation errors $\hat{\xi}$ and $\xi_{2}$, and therefore the estimation error $\hat{\xi}_{1}$, displayed in Fig. 3(b). The search for a minimum of $\hat{\xi}$ according to (8) for this example yields $T_{\text {opt }}=10$. In this case, a more accurate value for $\hat{T}_{\text {opt }}$ could have been obtained for a wider pipe, i.e. for a larger value of $K$.

While there are statistical approaches for hypothesis testing of ratios of variances such as the $F$-test [25], their application is currently unclear, as e.g. successive ratios $\gamma[T]$ in Fig. 3(a) are not independent. Therefore, $K$ is maintained as a variable, and we will explore different values for this parameter when assessing the approach in simulations in the following section. 


\section{Simulations, Results, ANd Discussion}

\section{A. Metrics}

Support Mismatch. Since the aim of the proposed approach is to determine the support of $\hat{\mathbf{R}}[\tau]$, a first performance metric is the support mismatch $\Delta T$ between the ideal and estimated values in (8) and (12),

$$
\Delta T=T_{\mathrm{opt}}-\hat{T}_{\mathrm{opt}}
$$

A positive value for $\Delta T$ means underestimation, $\Delta T<0$ overestimation of $T_{\mathrm{opt}}$. The effect of over- vs underestimation on the modelling error is unequal; e.g. Fig. 3 demonstrates that underestimation results generally in a greater modelling error $\hat{\xi}$ than overestimation. Therefore, the statistics of $\Delta T$ when averaged over an ensemble can be somewhat misleading.

Normalised Modelling Error Difference. Since analogously to the example in Fig. 3, the exact modelling error $\xi$ is known, we can find the minimum squared modelling error $\hat{\xi}_{\text {min }}$ for the optimum support $T_{\text {opt }}$ and the modelling error $\hat{\xi}_{\text {est }}$ obtained with the estimated support $\hat{T}_{\text {opt }}$,

$$
\begin{aligned}
\hat{\xi}_{\text {min }} & =\sum_{m, \mu, \tau}\left|r_{m \mu}[\tau]-\hat{r}_{m \mu}^{\left(T_{\text {opt }}\right)}[\tau]\right|^{2} \\
\hat{\xi}_{\text {est }} & =\sum_{m, \mu, \tau}\left|r_{m \mu}[\tau]-\hat{r}_{m \mu}^{\left(\hat{T}_{\text {opt }}\right)}[\tau]\right|^{2} .
\end{aligned}
$$

Normalising the difference such that

$$
\hat{\xi}_{\text {norm }}=\frac{\hat{\xi}_{\text {est }}-\hat{\xi}_{\text {min }}}{\sum_{m, \mu, \tau}\left|r_{m \mu}[\tau]\right|^{2}}
$$

creates a metric that is somewhat independent of a specific $\mathbf{R}[\tau]$, such that results become comparable across an ensemble of different realisations of $\mathbf{R}[\tau]$.

\section{B. Fixed Vs Variable Model}

The source model in [23] of order 30 has been used to provide a ground truth space-time covariance matrix $\mathbf{R}[\tau] \in$ $\mathbb{C}^{2 \times 2}$ and generate the data from which an estimate $\hat{\mathbf{R}}[\tau]$ can be built. In a first simulation, a fixed source model generates $10^{3}$ data sets, each of size $N=10^{3}$. Running the proposed approach with a variety of pipe widths for setting the threshold to identify $\hat{T}_{\text {opt }}$ provides a distribution of values for the metrics $\Delta T$ and $\hat{\xi}_{\text {norm }}$ that are presented in Fig. 4 . On average the best result for $\hat{\xi}_{\text {norm }}$ is achieved for $K \approx 8$, but the metrics do not appear to be too sensitive w.r.t. to this threshold.

For a randomised source model of order 30, also with $M=2$ and generating $N=10^{3}$ snapshots each, Fig. 5 shows the distribution of evaluated metrics over an ensemble of $10^{3}$ instantiations. The results are similar to those in Fig. 4 but with lower $\hat{\xi}$; to exclude the impact of 'unlucky' (or potentially in other cases 'lucky') picks in terms of a source model, the remaining simulations are performed using a randomised source model.
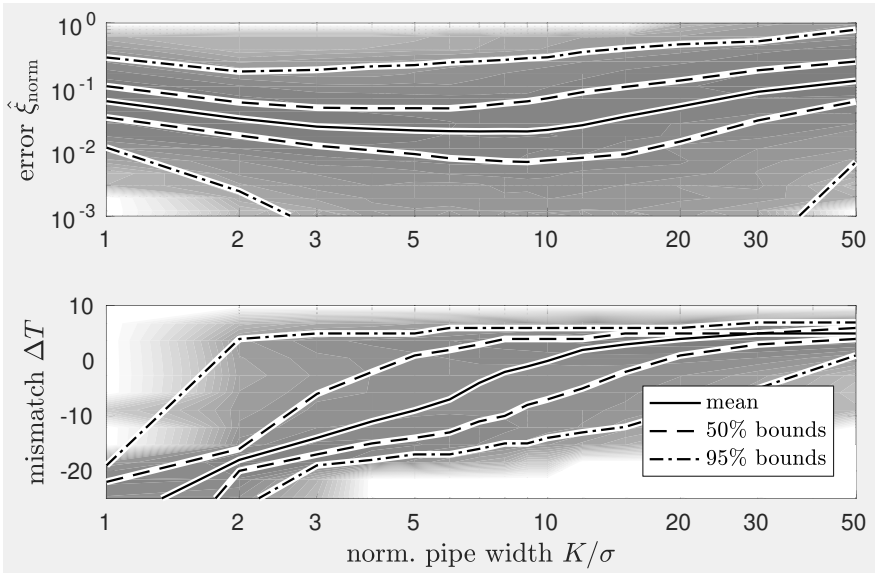

Fig. 4. Ensemble metrics for fixed model with $M=2$ and $N=10^{3}$.
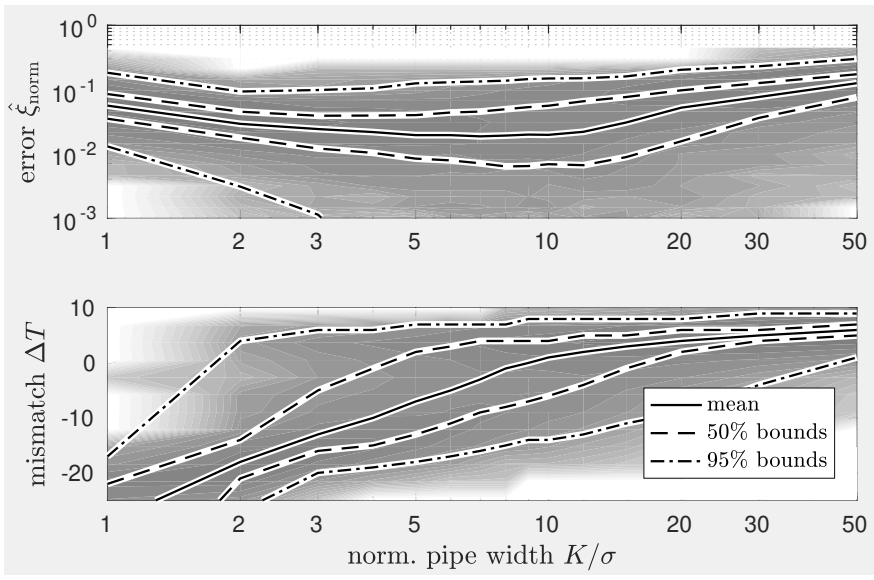

Fig. 5. Ensemble metrics for variable model, with $M=2, N=10^{3}$.

\section{Impact of Spatial Dimension}

When increasing the spatial dimension from $M=2$ to $M=6$, it might be expected that the summation over an increased number of terms in (10) and (11) leads to an enhanced averaging of $\gamma[T]$, with a possible reduction of the pipe width $K \sigma$ for a decision threshold. However, the results over an ensemble of 1000 source models in Fig. 6 suggest no change in the statistics of the metrics, and a threshold of $K \approx 8$ provides the lowest average modelling error.

\section{Impact of Sample Size}

Fig. 7 shows the results for an ensemble of $10^{3}$ source models for $M=2$ when increasing the sample size to $N=10^{4}$. In this case, the variance of the estimate is reduced, thus also reducing the modelling error. Note that in general, the modelling error drops by almost an order of magnitude compared to the case $\left\{M=2, N=10^{3}\right\}$ in Fig. 5. With a lower variance of the estimates, the ratio $\gamma_{1}[T]$ is also stabilised, such that in average the lowest modelling error is achieved for $K \approx 4$, i.e. with half the pipe width compared to the cases of $N=10^{3}$. 

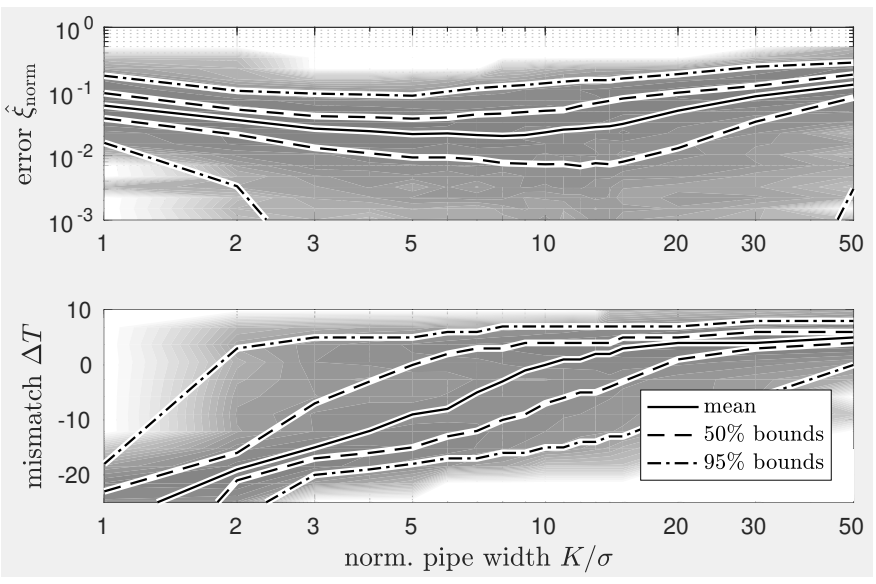

Fig. 6. Ensemble metrics for variable model, with $M=6, N=10^{3}$.
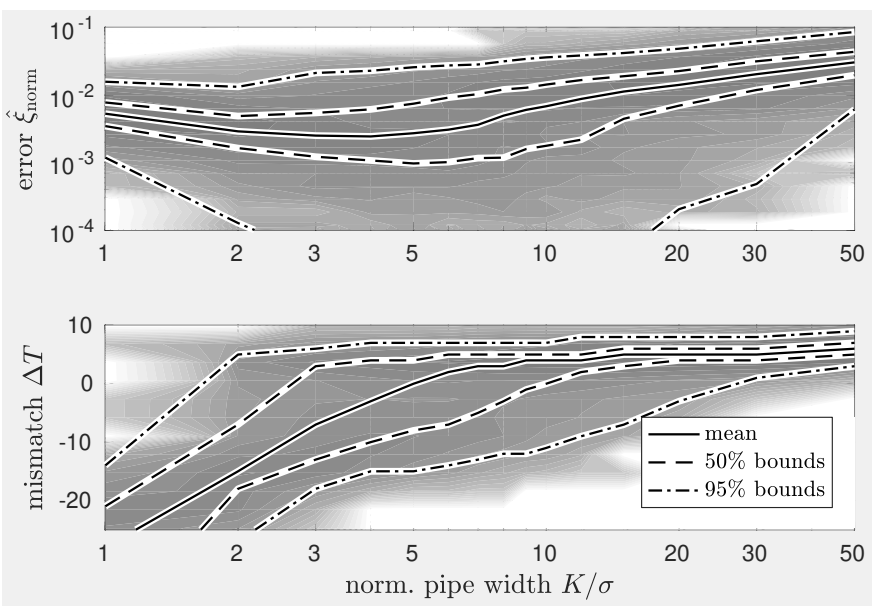

Fig. 7. Ensemble metrics for variable model, with $M=2, N=10^{4}$.

Overall, this demonstrates that the selection of $K$ is not too sensitive, but that an optimisation of the threshold for at least different sample sizes $N$ need to be made.

\section{CONCLUSIONS}

Based on previous exact analysis of the variance of sample space-time covariance matrix estimation, this paper has presented an empirical approach to the estimation of the support for such matrices. Inaccessible quantities such as the exact space-time covariance matrix, on which an optimum support selection would be based, are replaced by estimated quantities as approximations. A drawback of the proposed approach requires the computation of estimates of the space time covariance matrix over substantially more lags than will ultimately be selected as support. Also, a statisticallymotivated determination of the threshold has not been derived yet.

Nonetheless, simulation results demonstrate reasonable performance in terms of the achieved modelling error, and some insensitivity to the precise selection of the decision threshold for the estimated support, compared to what is achievable by with an optimum support selection based on knowledge of the ground truth.

\section{REFERENCES}

[1] C. H. Ta and S. Weiss, "A jointly optimal precoder and block decision feedback equaliser design with low redundancy," in EUSIPCO, Poznan, Poland, Sep. 2007, pp. 489-492.

[2] S. Weiss, S. Redif, T. Cooper, C. Liu, P. Baxter, and J. McWhirter, "Paraunitary oversampled filter bank design for channel coding," EURASIP Journal on Advances in Signal Processing, vol. 2006, pp. 1-10, 2006.

[3] S. Redif, J. McWhirter, P. Baxter, and T. Cooper, "Robust broadband adaptive beamforming via polynomial eigenvalues," in OCEANS, Boston, MA, Sep. 2006, pp. 1-6.

[4] S. Weiss, S. Bendoukha, A. Alzin, F. Coutts, I. Proudler, and J. Chambers, "MVDR broadband beamforming using polynomial matrix techniques," in EUSIPCO, Nice, France, Sep. 2015, pp. 839-843.

[5] S. Redif, S. Weiss, and J. McWhirter, "Relevance of polynomial matrix decompositions to broadband blind signal separation," Signal Processing, vol. 134, pp. 76-86, May 2017.

[6] S. Weiss, M. Alrmah, S. Lambotharan, J. McWhirter, and M. Kaveh, "Broadband angle of arrival estimation methods in a polynomial matrix decomposition framework," in IEEE CAMSAP, St. Martin, Dec. 2013, pp. $109-112$.

[7] S. Weiss, N. J. Goddard, S. Somasundaram, I. K. Proudler, and P. A. Naylor, "Identification of broadband source-array responses from sensor second order statistics," in SSPD, London, UK, Dec. 2017, pp. 1-5.

[8] J. G. McWhirter, P. D. Baxter, T. Cooper, S. Redif, and J. Foster, "An EVD Algorithm for Para-Hermitian Polynomial Matrices," IEEE Trans. Signal Processing, vol. 55, no. 5, pp. 2158-2169, May 2007.

[9] S. Weiss, J. Pestana, and I. K. Proudler, "On the existence and uniqueness of the eigenvalue decomposition of a parahermitian matrix," IEEE Trans. Signal Processing, vol. 66, no. 10, pp. 2659-2672, May 2018.

[10] S. Weiss, J. Pestana, I. Proudler, and F. Coutts, "Corrections to 'On the existence and uniqueness of the eigenvalue decomposition of a parahermitian matrix'," IEEE Trans. Signal Processing, vol. 66, no. 23 , pp. 6325-6327, Dec. 2018.

[11] J.G. McWhirter, "An algorithm for polynomial matrix SVD based on generalised Kogbetliantz transformations," in EUSIPCO, Aalborg, Denmark, Aug. 2010, pp. 457-461.

[12] G.H. Golub and C.F. Van Loan, Matrix Computations, 3rd ed. John Hopkins University Press, 1996.

[13] G. W. Stewart, "The decompositional approach to matrix computation," Computing in Science Engineering, vol. 2, no. 1, pp. 50-59, Jan. 2000.

[14] M. Alrmah, J. Corr, A. Alzin, K. Thompson, and S. Weiss, "Polynomial subspace decomposition for broadband angle of arrival estimation," in SSPD, Edinburgh, Scotland, Sep. 2014, pp. 1-5.

[15] F. Coutts, K. Thompson, S. Weiss, and I. Proudler, "Impact of fastconverging PEVD algorithms on broadband AoA estimation," in SSPD, London, UK, Dec. 2017, pp. 1-5.

[16] J. Foster, J. G. McWhirter, and J. Chambers, "Limiting the order of polynomial matrices within the SBR2 algorithm," in IMA Int. Conf. Math. in Signal Processing, Cirencester, UK, Dec. 2006.

[17] C. H. Ta and S. Weiss, "Shortening the order of paraunitary matrices in SBR2 algorithm," in IEEE ICICSP, Singapore, Dec. 2007, pp. 1-5.

[18] J. Corr, K. Thompson, S. Weiss, I. Proudler, and J. McWhirter, "Rowshift corrected truncation of paraunitary matrices for PEVD algorithms," in EUSIPCO, Nice, France, Aug. 2015, pp. 849-853.

[19] _ - "Shortening of paraunitary matrices obtained by polynomial eigenvalue decomposition algorithms," in SSPD, Edinburgh, Scotland, Sept. 2015.

[20] - "Impact of source model matrix conditioning on PEVD algorithms," in IET/EURASIP ISP, London, UK, Dec. 2015.

[21] C. Delaosa, J. Pestana, N. J. Goddard, S. Somsundaram, and S. Weiss, "Sample space-time covariamce matrix estimation," in IEEE ICASSP, Brighton, UK, May 2019.

[22] C. Delaosa, F. K. Coutts, J. Pestana, and S. Weiss, "Impact of spacetime covariance estimation errors on a parahermitian matrix EVD," in IEEE SAM, Sheffield, UK, July 2018, pp. 1-5.

[23] S. Redif, S. Weiss, and J. McWhirter, "Sequential matrix diagonalization algorithms for polynomial EVD of parahermitian matrices," IEEE Trans. Signal Processing, vol. 63, no. 1, pp. 81-89, Jan. 2015.

[24] P. J. Schreier and L. L. Scharf, Statistical signal processing of complexvalued data. The theory of improper and non-circular signals. Cambridge University Press, 2010.

[25] I. Bronshtein and K. Semendyayew, Handbook of Mathematics. Heidelberg: Springer, 2015. 